Pacific Journal of Mathematic 


\title{
CONVOLUTION TRANSFORMS WITH COMPLEX KERNELS
}

\author{
I. I. Hirschman, JR. AND D. V. Widder
}

1. Introduction. In the present paper we shall consider the inversion of a class of convolution transforms with kernel $G(t)$ of the form

$$
\begin{array}{ccc}
G(t)=\frac{1}{2 \pi i} \int_{-i \infty}^{i \infty}[E(s)]^{-1} e^{s t} d s & (-\infty<t<\infty), \\
E(s)=\prod_{1}^{\infty}\left(1-\frac{s}{a_{k}}\right) e^{s / b_{k}}, &
\end{array}
$$

$a_{k}=b_{k}+i c_{k}(k=1,2, \cdots)$ being a sequence of complex numbers such that

$$
\sum_{k=1}^{\infty}\left(1 / b_{k}\right)^{2}<\infty, \quad \sum_{k=1}^{\infty}\left(c_{k} / b_{k}\right)^{2}<\infty .
$$

This class of kernels is more extensive than that treated previously by the authors, see $[4],[5],[6]$, and $[7]$; however the results obtained here are slightly less precise than those which it was possible to obtain there. We shall show essentially that if

$$
f(x)=\int_{-\infty}^{\infty} G(x-t) d \alpha(t)
$$

and if $x_{1}$ and $x_{2}$ are points of continuity of $\alpha(t)$, then

$$
\lim _{m \rightarrow \infty} \int_{x_{1}}^{x_{2}}\left[\prod_{k=1}^{m}\left(1-\frac{D}{a_{k}}\right) e^{D / b_{k}}\right] f(x) d x=\alpha\left(x_{2}\right)-\alpha\left(x_{1}\right) .
$$

Here $D$ is the operation of differentiation, and $e^{D / a}$ that of translation through the

Pacific J. Math. 1 (1951), 211-225. 
distance $1 / a$, so that, for example,

$$
\begin{aligned}
\left(1-\frac{D}{a_{1}}\right) e^{D / b_{1}}\left(1-\frac{D}{a_{2}}\right) e^{D / b_{2}} f(x)=f\left(x+\frac{1}{b_{1}}+\frac{1}{b_{2}}\right) & -\left(\frac{1}{a_{1}}+\frac{1}{a_{2}}\right) f^{\prime}\left(x+\frac{1}{b_{1}}+\frac{1}{b_{2}}\right) \\
& +\frac{1}{a_{1} a_{2}} f^{\prime \prime}\left(x+\frac{1}{b_{1}}+\frac{1}{b_{2}}\right) .
\end{aligned}
$$

If we replace equation (1.2) and inequalities (1.3) by the more special relations

$$
E(s)=\prod_{k=1}^{\infty}\left(1-\frac{s^{2}}{a_{k}^{2}}\right)
$$

$$
\lim _{k \rightarrow \infty} b_{k} / k=\Omega>0, \quad \sum_{k=1}^{\infty}\left(c_{k} / b_{k}\right)^{2}<\infty,
$$

we have in addition the complex inversion formula,

$$
\lim _{\lambda \rightarrow 1^{-}} \int_{x_{1}}^{x_{2}} d x \frac{1}{2 \pi i} \int_{C_{\lambda}} f(\lambda w+x) K(w) d w=\alpha\left(x_{2}\right)-\alpha\left(x_{1}\right),
$$

where

$$
K(w)=\int_{0}^{\infty} E(s) e^{-s w} d s
$$

and $C_{\lambda}$ is a closed rectifiable curve encircling the segment $[-i \Omega, i \Omega]$ and lying in the strip $|d w|<\Omega / \lambda$. The inner integral in formula (1.8) is to be taken in the counterclockwise direction.

As one example we may take

$$
\begin{aligned}
E(s) & =\frac{\Gamma(1 / 2+\nu / 2)^{2}}{\Gamma(1 / 2+\nu / 2-s / 2) \Gamma(1 / 2+\nu / 2+s / 2)}, \\
G(t) & =\frac{\left(e^{t}+e^{-t}\right)^{-\nu-1}}{\Gamma(1 / 2+\nu / 2)^{2}},
\end{aligned}
$$


where $R_{\nu}>-1$. If

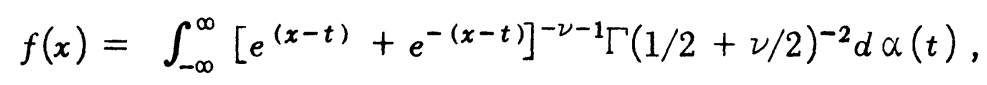

then

$$
\lim _{m \rightarrow \infty} \int_{x_{1}}^{x_{2}}\left\{\prod_{k=1}^{m}\left[1-\left(\frac{D}{-1 / 2+\nu / 2+k}\right)^{2}\right] f(x)\right\} d x=\alpha\left(x_{2}\right)-\alpha\left(x_{1}\right) ;
$$

and if $R_{\nu}>0$, then

$$
\begin{array}{r}
\lim _{\lambda \rightarrow 1^{-}} \frac{2^{\nu} \Gamma(1 / 2+\nu / 2)^{2}}{\pi \Gamma(\nu)} \int_{x_{1}}^{x_{2}} d x \int_{-\pi / 2}^{\pi / 2} f(x+i \lambda w)[\cos w]^{\nu-1} d w \\
=\alpha\left(x_{2}\right)-\alpha\left(x_{1}\right) .
\end{array}
$$

See [7] and [8], and [9]. A second example is

$$
\begin{aligned}
& E(s)=\pi 2^{s}\left[\cos \frac{\pi \nu}{2} \Gamma\left(\frac{1}{2}-\frac{\nu}{2}-\frac{s}{2}\right) \Gamma\left(\frac{1}{2}+\frac{\nu}{2}-\frac{s}{2}\right)\right]^{-1}, \\
& G(t)=\frac{2}{\pi} \cos \frac{\pi \nu}{2} e^{t} K_{\nu}\left(e^{t}\right)
\end{aligned}
$$

for $-1<R_{\nu}<1$. If

$$
f(x)=\int_{-\infty}^{\infty} e^{x-t} K_{\nu}\left(e^{x-t}\right)\left(\frac{2}{\pi} \cos \frac{\nu \pi}{2}\right) d \alpha(t),
$$

then

$$
\begin{array}{r}
\lim _{m \rightarrow \infty} \int_{x_{1}}^{x_{2}}\left\{\prod_{k=1}^{m}\left(1-\frac{D}{-1 / 2-\nu / 2+k}\right)\left(1-\frac{D}{-1 / 2+\nu / 2+k}\right) f(x)\right\} d x \\
=\alpha\left(x_{2}\right)-\alpha\left(x_{1}\right) .
\end{array}
$$

See [2].

2. Inversion of a class of convolution transforms. We assume as given throughout this section a sequence, $\left\{a_{k}\right\}_{1}^{\infty}$, of complex numbers $a_{k}=b_{k}+i c_{k}$ subject 
to the restrictions

$$
\sum_{k=1}^{\infty}\left(1 / b_{k}\right)^{2}<\infty, \quad \sum_{k=1}^{\infty}\left(c_{k} / b_{k}\right)^{2}<\infty .
$$

We define the entire functions

$$
\begin{aligned}
E_{m, n}(s) & =\prod_{k=m+1}^{n}\left(1-s / a_{k}\right) e^{s / b_{k}}, \\
E_{m}(s) & =\prod_{k=m+1}^{\infty}\left(1-s / a_{k}\right) e^{s / b_{k}}, \\
F_{m}(s) & =\prod_{k=m+1}^{\infty}\left|b_{k} / a_{k}\right|\left(1-s / b_{k}\right) e^{s / b_{k}} .
\end{aligned}
$$

The definition of $E_{m}(s)$ is significant because

$$
E_{m}(s)=\left\{\prod_{m+1}^{\infty}\left(1-\frac{s}{a_{k}}\right) e^{s / a_{k}}\right\}\left\{\exp \sum_{m+1}^{\infty} \frac{i c_{k} s}{b_{k}\left(b_{k}+i c_{k}\right)}\right\},
$$

and because the series $\sum_{m+1}^{\infty}\left|a_{k}\right|^{-2}, \sum_{m+1}^{\infty} c_{k} / b_{k}\left(b_{k}+i c_{k}\right)$ converge as a consequence of (2.1) and Schwarz's inequality. Similarly, $F_{m}(s)$ is well defined. We define

$$
P_{m}(D)=\prod_{k=1}^{m}\left(1-D / a_{k}\right) e^{D / b_{k}} \quad(m=0,1, \cdots)
$$

We also set

$$
\beta_{1}(m)=\max _{\substack{b_{k}<0 \\ k>m}}\left(b_{k},-\infty\right), \quad \beta_{2}(m)=\min _{\substack{b_{k}>0 \\ k>m}}\left(b_{k}, \infty\right),
$$

THEOREM 2a. Let

$$
G_{m}(t)=\frac{1}{2 \pi i} \int_{-i \infty}^{i \infty}\left[E_{m}(s)\right]^{-1} e^{s t} d s \quad(-\infty<t<\infty ; m=0,1,2, \cdots) ;
$$


then we have
A. $\int_{-\infty}^{\infty} G_{m}(t) e^{-s t} d t=1 / E_{m}(s)$, $\beta_{1}(m)<R_{s}<\beta_{2}(m) ;$
B. $\int_{-\infty}^{\infty}\left|G_{m}(t)\right| e^{-\sigma t} d t \leq 1 / F_{m}(\sigma)$, $\beta_{1}(m)<\sigma<\beta_{2}(m) ;$
C. $\quad P_{m}(D) G_{0}(t)=G_{m}(t)$;
D. $(d / d t)^{k} G_{m}(t)=O\left(e^{\gamma_{1} t}\right), t \rightarrow+\infty$,

$$
=O\left(e^{\gamma_{2} t}\right), t \rightarrow-\infty
$$$$
(k=0,1, \cdots),
$$

for $\gamma_{1}>\beta_{1}(m)$ and $\gamma_{2}<\beta_{2}(m)$.

Conclusion $\mathrm{A}$ is an immediate consequence of Hamburger's theorem; see [4, pp.141-144]. We define $g(u)=e^{u-1}$ for $-\infty<u \leq 1$, and $g(u)=0$ for $1<u<\infty$, and we set

$$
g_{k}(t)=a_{k} \operatorname{sgn} b_{k}\left\{\exp \left[i c_{k}\left(t-b_{k}^{-1}\right)\right]\right\} g\left(b_{k} t\right) .
$$

It is immediately verifiable that

$$
\int_{-\infty}^{\infty} e^{-s t} g_{k}(t) d t=\left[\left(1-\frac{s}{a_{k}}\right) e^{s / b_{k}}\right]^{-1},
$$

for $-\infty<R_{s}<b_{k}$ if $b_{k}>0$, and for $b_{k}<R_{s}<\infty$ if $b_{k}<0$. Let

$$
g_{1} * g_{2}(t)=\int_{-\infty}^{\infty} g_{1}(t-u) g_{2}(u) d u,
$$

and so on; then by the convolution theorem for the bilateral Laplace transform we have

$$
\int_{-\infty}^{\infty} g_{m+1} * g_{m+2} * \cdots * g_{n}(t) e^{-s t} d t=\left[E_{m, n}(s)\right]^{-1}
$$

for $\beta_{1}(m)<R_{s}<\beta_{2}(m)$. From the complex inversion formula for the bilateral Laplace transform we obtain

$$
g_{m+1} * g_{m+2} * \cdots * g_{n}(t)=\frac{1}{2 \pi i} \int_{-i \infty}^{i \infty}\left[E_{m, n}(s)\right]^{-1} e^{s t} d s
$$


Since

$$
\lim _{n \rightarrow \infty} \frac{1}{2 \pi i} \int_{-i \infty}^{i \infty}\left\{\left[E_{m}(s)\right]^{-1}-\left[E_{m, n}(s)\right]^{-1}\right\} e^{s t} d s=0
$$

for $-\infty<t<\infty$, it follows that

$$
\lim _{n \rightarrow \infty} g_{m+1} * \cdots * g_{n}(t)=G_{m}(t) \quad(-\infty<t<\infty) \text {. }
$$

See $[4 ; \mathrm{pp} .139-145]$. It is easily seen that

$$
\int_{-\infty}^{\infty}\left|g_{k}(t)\right| e^{-s t} d t=\left[\left(1-s / b_{k}\right) e^{s / b_{k}}\left|b_{k} / a_{k}\right|\right]^{-1}
$$

for $-\infty<R_{s}<b_{k}$ if $b_{k}>0$, or for $b_{k}<R s<\infty$ if $b_{k}<0$. By Fatou's lemma we have

$$
\begin{aligned}
\int_{-\infty}^{\infty}\left|G_{m}(t)\right| e^{-\sigma t} d t & \leq \liminf _{n \rightarrow \infty} \int_{-\infty}^{\infty}\left|g_{m+1} * \cdots * g_{n}(t)\right| e^{-\sigma t} d t \\
& \leq \liminf _{n \rightarrow \infty} \int_{-\infty}^{\infty}\left|g_{m+1}\right| * \cdots *\left|g_{n}(t)\right| e^{-\sigma t} d t \\
& \leq\left[F_{m}(\sigma)\right]^{-1}
\end{aligned}
$$

so that conclusion $B$ is established.

Conclusion $\mathrm{C}$ follows from the identity

$$
P_{m}(D) e^{s t}=e^{s t} \prod_{k=1}^{m}\left(1-s a_{k}^{-1}\right) e^{s / a_{k}}
$$

Conclusion $D$ may be established by shifting the line of integration in the integral defining $G_{m}(t)$ to $R_{s}=\gamma_{1}$ and $R_{s}=\gamma_{2}$. See [4; pp. 152-154].

In what follows we shall write $G(t)$ for $G_{0}(t)$.

THEOREM $2 \mathrm{~b}$. If

(a) $G(t)$ is defined as in Theorem $2 \mathrm{a}$,

(b) $\beta_{1}(0)<c<\beta_{2}(0), c+\gamma_{1}>\beta_{1}(0), c+\gamma_{2}<\beta_{2}(0)$, 
(c) $\quad \alpha(t)$ is of bounded variation on every finite interval, $\alpha(t)=O\left(e^{\gamma_{1} t}\right)$ as $t \rightarrow-\infty, \alpha(t)=O\left(e^{\gamma_{2} t}\right)$ as $t \rightarrow+\infty$,

(d) $\quad P_{m}(D)$ is defined as in equation (2.3),

(e) $f(x)=\int_{-\infty}^{\infty} G(x-t) e^{c t} d \alpha(t)$,

(f) $\quad x_{1}$ and $x_{2}$ are points of continuity of $\alpha(t)$, then

$$
\lim _{m \rightarrow \infty} \int_{x_{1}}^{x_{2}} e^{-c x}\left[P_{m}(D) f(x)\right] d x=\alpha\left(x_{2}\right)-\alpha\left(x_{1}\right)
$$

From assumption (c) and from conclusion D of Theorem 2a we may show, using integration by parts, that each of the integrals

$$
\int_{-\infty}^{\infty} G_{m}(x-t) e^{c t} d \alpha(t)
$$

converges uniformly for $x$ in any finite interval. Since $P_{m}(D) G(t)=G_{m}(t)$ by conclusion $\mathrm{C}$ of Theorem 2a, it follows (see [4; pp. 167-170]) that

$$
P_{m}(D) f(x)=\int_{-\infty}^{\infty} G_{m}(x-t) e^{c t} d \alpha(t) \quad(-\infty<x<\infty) .
$$

Multiplying by $e^{-c x}$ and integrating by parts, we have

$$
\begin{aligned}
e^{-c x} P_{m}(D) f(x) & =-\int_{-\infty}^{\infty}\left\{\frac{\partial}{\partial t}\left[G_{m}(x-t) e^{-c(x-t)}\right]\right\} \alpha(t) d t \\
& =\int_{-\infty}^{\infty}\left\{\frac{\partial}{\partial x}\left[G_{m}(x-t) e^{-c(x-t)}\right]\right\} \alpha(t) d t .
\end{aligned}
$$

Since this integral converges uniformly for $x$ in any finite interval, we obtain

$$
\begin{aligned}
\int_{x_{1}}^{x_{2}} & e^{-c x} P_{m}(D) f(x) d x \\
= & \int_{x_{1}}^{x_{2}} d x \quad \int_{-\infty}^{\infty}\left\{\frac{\partial}{\partial x}\left[G_{m}(x-t) e^{-c(x-t)}\right]\right\} \alpha(t) d t \\
= & \int_{-\infty}^{\infty} \alpha(t) d t \quad \int_{x_{1}}^{x_{2}}\left\{\frac{\partial}{\partial x}\left[G_{m}(x-t) e^{-c(x-t)}\right]\right\} d x
\end{aligned}
$$




$$
\begin{aligned}
& =\int_{-\infty}^{\infty}\left\{G_{m}\left(x_{2}-t\right) e^{-c\left(x_{2}-t\right)}-G_{m}\left(x_{1}-t\right) e^{-c\left(x_{1}-t\right)}\right\} \alpha(t) d t \\
& =\int_{-\infty}^{\infty} G_{m}\left(x_{2}-t\right) e^{-c\left(x_{2}-t\right)} \alpha(t) d t-\int_{-\infty}^{\infty} G_{m}\left(x_{1}-t\right) e^{-c\left(x_{1}-t\right)} \alpha(t) d t .
\end{aligned}
$$

We thus need only show that if $x$ is a point of continuity of $\alpha(t)$ we have

$$
\lim _{m \rightarrow \infty} \int_{-\infty}^{\infty} G_{m}(x-t) e^{-c(x-t)} \alpha(t) d t=\alpha(x) .
$$

We shall first show that for any $\epsilon>0$ we have

$$
\lim _{m \rightarrow \infty} \int_{|t| \geq \epsilon} G_{m}(t) e^{-c t} \alpha(x-t) d t=0 .
$$

Using assumptions (a) and (b) we see that it is enough to prove that for any $\delta$ with $\beta_{1}(0)<\delta<\beta_{2}(0)$, we have

$$
\lim _{m \rightarrow \infty} \int_{|t| \geq \epsilon}\left|G_{m}(t)\right| e^{-\delta t} d t=0
$$

Choose $\eta>0$ so small that $\beta_{1}(0)<\delta-2 \eta<\delta+2 \eta<\beta_{2}(0)$. For $|t| \geq \epsilon$ we have

$$
e^{-\delta t} \leq \frac{e^{-\delta t}(\sinh \eta t)^{2}}{(\sinh \epsilon \eta)^{2}}
$$

so that it is enough to prove that

$$
\lim _{m \rightarrow \infty} \int_{-\infty}^{\infty}\left|G_{m}(t)\right| e^{-\delta t}[\sinh \eta t]^{2} d t=0
$$

Using conclusions $\mathrm{A}$ and $\mathrm{B}$ of Theorem $2 \mathrm{a}$ we see that

$$
\begin{aligned}
\int_{-\infty}^{\infty}\left|G_{m}(t)\right| e^{-\delta t}[\sinh \eta t]^{2} d t \\
\quad \leq \frac{1}{4}\left[\frac{1}{F_{m}(\delta+2 \eta)}+\frac{1}{F_{m}(\delta-2 \eta)}-\frac{2}{E_{m}(\delta)}\right]=o(1) \quad(m \rightarrow+\infty),
\end{aligned}
$$

and equation (2.8) follows from this. We assert that

$$
\lim _{m \rightarrow \infty} \int_{-\infty}^{\infty} G_{m}(t) e^{-c t} d t=1
$$




$$
\lim _{m \rightarrow \infty} \int_{-\infty}^{\infty}\left|G_{m}(t)\right| e^{-c t} d t=1
$$

These results are immediate consequences of conclusions $\mathrm{A}$ and $\mathrm{B}$ of Theorem $2 \mathrm{a}$. Now $x$ being fixed and $\eta>0$ being given, let us choose $\epsilon>0$ so small that $|\alpha(t)-\alpha(x)| \leq \eta$ for $|t-x| \leq \epsilon$. We have

$$
\int_{-\infty}^{\infty} G_{m}(x-t) e^{-c(x-t)} \alpha(t) d t-\alpha(x)=I_{1}+I_{2}+I_{3},
$$

where

$$
\begin{aligned}
& I_{1}=\alpha(x)\left[\int_{-\infty}^{\infty} G_{m}(x-t) e^{-c(x-t)} d t-1\right] \\
& I_{2}=\int_{|t| \geq \epsilon} G_{m}(t) e^{-c t}[\alpha(x-t)-\alpha(x)] d t \\
& I_{3}=\int_{|t| \leq \epsilon} G_{m}(t) e^{-c t}[\alpha(x-t)-\alpha(x)] d t .
\end{aligned}
$$

We have $\lim _{m \rightarrow \infty} I_{1}=0$ by equation (2.9), $\lim _{m \rightarrow \infty} I_{2}=0$ by equation (2.7), and $\lim \sup _{m \rightarrow \infty}\left|I_{3}\right| \leq \eta$ by equation (2.10). Since $\eta$ is arbitrary our demonstration is complete.

3. Complex inversion formulas. In this section we restrict our attention to a much more special class of kernels. We suppose that

$$
b_{k}>0, \quad b_{k} \sim \frac{\Omega k}{\pi}, \quad \sum_{k=1}^{\infty}\left(\frac{c_{k}}{b_{k}}\right)^{2}<\infty .
$$

We define

$$
\begin{gathered}
E(s)=\prod_{k=1}^{\infty}\left(1-\frac{s}{a_{k}^{2}}\right)^{2}, \\
H(\lambda, s)=\prod_{k=1}^{\infty}\left[\lambda^{2}+\left(1-\lambda^{2}\right) \frac{\left|a_{k}\right| b_{k}}{b_{k}^{2}-s^{2}}\right] \quad(0 \leq \lambda<1) .
\end{gathered}
$$


The product (3.3) is defined for $s \neq b_{k}(k=1,2, \cdots)$ since it can be rewritten as

$$
H(\lambda, s)=\frac{\prod_{k=1}^{\infty}\left[1-\frac{\lambda^{2} s^{2}}{b_{k}^{2}}+\left(1-\lambda^{2}\right) \frac{\left|a_{k}\right|-b_{k}}{b_{k}}\right]}{\prod_{k=1}^{\infty}\left(1-\frac{s^{2}}{b_{k}^{2}}\right)},
$$

and assumption (a) implies that $\Sigma_{1}^{\infty}\left[\left|a_{k}\right|-b_{k}\right] b_{k}^{-1}$ is convergent. We define

$$
\beta=\min b_{k}
$$

THEOREM 3a. If

$$
G(\lambda, w)=\frac{1}{2 \pi i} \quad \int_{-i \infty}^{i \infty} \frac{e^{s w} E(\lambda s)}{E(s)} d s \quad(0 \leq \lambda<1)
$$

then

A. $\quad G(\lambda, w)$ is analytic for $|d w|<\Omega(1-\lambda)$;

B. $\quad \int_{-\infty}^{\infty} G(\lambda, t) e^{-s t} d t=E(\lambda s) / E(s),-\beta<R s<\beta$;

C. $(d / d w)^{k} G(\lambda, w)=0\left(e^{\gamma_{1} u}\right) \quad(u \rightarrow+\infty)$

$$
=0\left(e^{\gamma 2 u}\right)(u \rightarrow-\infty) \quad(k=0,1, \cdots),
$$

where $\gamma_{1}>-\beta, \gamma_{2}<\beta$, uniformly for $|v|<\Omega(1-\lambda)-\epsilon, \epsilon>0$. (Here $w=u+i v$.

D. $\quad \int_{-\infty}^{\infty}|G(\lambda, t)| e^{-\sigma t} d t \leq H(\lambda, \sigma), \quad-\beta<\sigma<\beta$.

We shall write $G(t)$ for $G(0, t)$.

We assert that 


$$
\log |E(\sigma+i \tau)| \sim \Omega|\tau|
$$

uniformly for $\sigma$ in any finite interval. We define

$$
E *(s)=\prod_{k=1}^{\infty}\left(1-s^{2} b_{k}^{-2}\right) .
$$

We have

$$
\frac{E(s)}{E *(s)}=\prod_{k=1}^{\infty} \frac{a_{k}^{2}}{b_{k}^{2}} \prod_{k=1}^{\infty}\left\{1+\frac{a_{k}^{2}-b_{k}^{2}}{b_{k}^{2}-s^{2}}\right\},
$$

from which it follows that

$$
\lim _{s \rightarrow \infty} \frac{E(s)}{E *(s)}=\prod_{k=1}^{\infty} \frac{a_{k}^{2}}{\dot{b}_{k}^{2}}
$$

uniformly for $0<\epsilon \leq|\arg s| \leq \pi-\epsilon$. From [1, pp. 267-279] we have that $\log |E *(\sigma+i \tau) \sim \Omega| \tau \mid$ as $\tau \rightarrow \pm \infty$, uniformly for $\sigma$ in any finite interval. Relation (3.5) now follows.

Conclusion A follows immediately from (3.5) and the definition of $G(\lambda, w)$. Conclusion B is a consequence of (3.5) and Hamburger's Theorem. The two conclusions $\mathrm{C}$ are obtained by shifting the line of integration in the integral defining $G(\lambda, t)$ to $R_{s}=\gamma_{1}$, and to $R_{s}=\gamma_{2}$, respectively. See [6, pp. 688-691]. To establish conclusion $D$ we introduce the functions

$$
G_{n}(\lambda, t)=\frac{1}{2 \pi i} \ddot{\int}_{-i \infty}^{i \infty} e^{s t} \frac{\prod_{k=1}^{n}\left(1-\frac{\lambda^{2} s^{2}}{a_{k}^{2}}\right)}{\prod_{k=1}^{\infty}\left(1-\frac{s^{2}}{a_{k}^{2}}\right)} d s
$$

It is immediate that

$$
\lim _{n \rightarrow \infty} G_{n}(\lambda, t)=G(\lambda, t) \quad(-\infty<t<\infty) .
$$


We define

$$
h_{k}(\lambda, t)=\lambda^{2} j(t)+\left(1-\lambda^{2}\right) \frac{a_{k}}{2} \int_{-\infty}^{t} e^{-a_{k}|u|} d u
$$

where $j(t)=0$ for $-\infty<t<0 ; j(0)=1 / 2 ; j(t)=1$ for $0<t<\infty$. It is easily verified that for $-b_{k}<\sigma<b_{k}$ we have

$$
\int_{-\infty}^{\infty} e^{-s t} d h_{k}(\lambda, t)=\frac{1-\lambda^{2} s^{2} / a_{k}^{2}}{1-s^{2} / a_{k}^{2}}
$$

Just as in $\$ 2$ we may show that

$$
G_{n}(\lambda, t)=\lim _{m \rightarrow \infty} \frac{d}{d t}\left[h_{1}(\lambda, t) * \cdots * h_{n}(\lambda, t) * h_{n+1}(0, t) * \cdots * h_{m}(0, t)\right] .
$$

Here $h_{1} * h_{2}(t)=\int_{-\infty}^{\infty} h_{1}(t-u) d h_{1}(u)$. Note that this differs from the convention employed in $\$ 2$. By Fatou's lemma,

$$
\begin{aligned}
& \int_{-\infty}^{\infty} e^{-\sigma t}\left|G_{n}(\lambda, t)\right| d t
\end{aligned}
$$

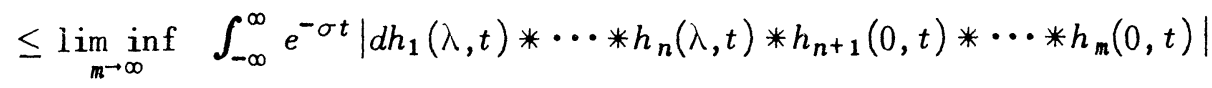

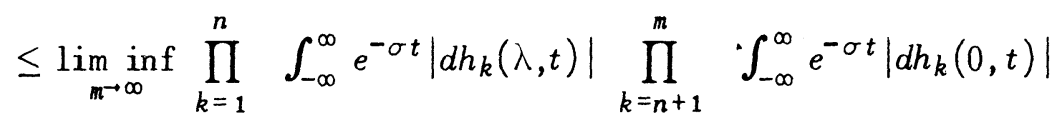

$$
\begin{aligned}
& \leq \prod_{k=1}^{n} \int_{-\infty}^{\infty} e^{-\sigma t}\left|d h_{k}(\lambda, t)\right| \prod_{k=n+1}^{\infty} \int_{-\infty}^{\infty} e^{-\sigma t}\left|d h_{k}(0, t)\right|
\end{aligned}
$$

By Fatou's lemma, again,

$$
\begin{aligned}
\int_{-\infty}^{\infty} e^{-\sigma t}|G(\lambda, t)| d t & \leq \liminf _{n \rightarrow \infty} \int_{-\infty}^{\infty} e^{-\sigma t}\left|G_{n}(\lambda, t)\right| d t \\
& \leq \prod_{k=1}^{\infty} \int_{-\infty}^{\infty} e^{-\sigma t}\left|d h_{k}(\lambda, t)\right| \\
& \leq \prod_{k=1}^{\infty}\left[\lambda^{2}+\left(1-\lambda^{2}\right) \frac{\left|a_{k}\right| b_{k}}{b_{k}^{2}-\sigma^{2}}\right] .
\end{aligned}
$$


This completes the proof of the theorem.

We define

$$
K(w)=\int_{0}^{\infty} E(s) e^{-s w} d s .
$$

It follows from relations (3.1) that given $\epsilon>0$, for all sufficiently large $r$ we have $\log \left|E *\left(r e^{i \theta}\right)\right| \leq(\epsilon+|\sin \theta|) \Omega r$. See [1, pp. 267-279]. From equation (3.6) it follows that

$$
\log \left|E\left(r e^{i \theta}\right)\right| \leq(\epsilon+|\sin \theta|) \Omega r
$$

for $r$ sufficiently large. Using this inequality and rotating the line of integration in the integral defining $K(w)$ we can show that $K(w)$ is analytic and single valued in the $w$-plane except on the segment $[-i \Omega, i \Omega]$. It may also be shown, see [1, pp. 295-311], that if $C$ is a closed rectifiable curve encircling $[-i \Omega, i \Omega]$ then

$$
E(s)=\frac{1}{2 \pi i} \int_{C} K(w) e^{s w} d w,
$$

the integration proceeding in the counterclockwise direction.

LEMMA $3 \mathrm{~b}$. If $C_{\lambda}$ is a closed rectifiable curve encircling $[-i \Omega, i \Omega]$ and contained in the strip $|v|<\Omega / \lambda$, then

$$
\frac{1}{2 \pi i} \int_{C_{\lambda}} G(\lambda w+x-t) K(w) d w=G(\lambda, x-t),
$$

the integration proceeding in the counterclockwise direction.

We have

$$
\begin{aligned}
\frac{1}{2 \pi i} \int_{C_{\lambda}} G(\lambda w+x-t) K(w) d w & \\
& =\frac{1}{2 \pi i} \int_{C_{\lambda}} K(w) d w \frac{1}{2 \pi i} \int_{-i \infty}^{i \infty}[E(s)]^{-1} e^{s(\lambda w+x-t)} d s \\
& =\frac{1}{2 \pi i} \int_{-i \infty}^{i \infty}[E(s)]^{-1} e^{s(x-t)} d s \frac{1}{2 \pi i} \int_{C_{\lambda}} K(w) e^{\lambda s w} d w
\end{aligned}
$$




$$
\begin{aligned}
& =\frac{1}{2 \pi i} \int_{-i \infty}^{i \infty}\left[\frac{E(\lambda s)}{E(s)}\right] e^{s(x-t)} d s \\
& =G(\lambda, x-t) .
\end{aligned}
$$

THEOREM 3c. If

(a) $G(t)$ is defined as in Theorem $3 \mathrm{a}$

(b) $-\beta<c<\beta,-\beta<c+\gamma_{1}, c+\gamma_{2}<\beta$

(c) $\quad \alpha(t)$ is of bounded variation on every finite interval and

$$
\alpha(t)=\left(e^{\gamma_{1} t}\right) \quad(t \rightarrow+\infty), \quad \alpha(t)=\left(e^{\gamma_{2} t}\right) \quad(t \rightarrow-\infty)
$$

(d) $f(w)=\int_{-\infty}^{\infty} G(w-t) e^{c t} d \alpha(t)$

(e) $K(w)$ is defined as in equation (3.7)

(f) $C_{\lambda}$ is defined as in Lemma $3 \mathrm{~b}$

(g) $\quad x_{1}$ and $x_{2}$ are points of continuity of $\alpha(t)$, then

$$
\lim _{\lambda \rightarrow 1-} \int_{x_{1}}^{x_{2}} e^{-c x} d x \frac{1}{2 \pi i} \int_{C \lambda} f(\lambda w+x) K(w) d w=\alpha\left(x_{2}\right)-a\left(x_{1}\right) .
$$

It follows from assumption (c) and from conclusion $\mathrm{C}$ of Theorem 3a that the integral defining $f(w)$ converges uniformly for $w$ in any compact set contained in the strip $|d w|<\Omega$. Hence

$$
\begin{aligned}
\frac{1}{2 \pi i} \int_{C_{\lambda}} & f(\lambda w+x) K(w) d w \\
& =\int_{-\infty}^{\infty} e^{c t} d a(t) \frac{1}{2 \pi i} \int_{C_{\lambda}} G(\lambda w+x-t) K(w) d w \\
& =\int_{-\infty}^{\infty} G(\lambda, x-t) e^{c t} d a(t)
\end{aligned}
$$

by Lemma $3 b$. The proof may now be completed exactly in the manner of Theorem $2 b$.

4. Remark. If it is assumed that the roots of $E(s)$ occur in conjugate pairs, then equation (1.5) can be established under conditions less restrictive than (1.3). A discussion of this case is given in the Master's thesis of Mr. A. O. Garder [3], written under the direction of one of us. 


\section{REFERENCES}

1. V. Bernstein, Leçons sur les progrès recents de la théorie des séries de Dirichlet, Paris, 1933.

2. R. P. Boas, Jr., Inversion of a generalized Laplace integral, Proc. Nat. Acad. Sci. U. S. A. 28 (1942), 21-24.

3. A. O. Garder, The inversion of a special class of convolution transforms, Master's Thesis, Washingt on University, 1950.

4. I. I. Hirschman, Jr. and D. V. Widder, The inversion of a general class of convolution transforms, Trans. Amer. Math. Soc. 66 (1949), 135-201.

5. - A representation theory for a general class of convolution transforms, Trans. Amer. Math. Soc. 67 (1949), 69-97.

6. J. 15 (1948), 659-696.

7. - Generalized inversion formulas for convolution transforms, II, Duke Math. J. 17 (1950), 391-402.

8. H. Pollard, Studies on the Stieltjes transform, Dissertation, Harvard; Abstract 48-3-117, Bull. Amer. Math. Soc. 48 (1942), 214.

9. D. B. Sumner, An inversion formula for the generalized Stieltjes transform, Bull. Amer. Math. Soc. 55 (1949), 174-183.

10. D. V. Widder, The Laplace transform, Princeton University Press, Princeton, 1941.

\section{WASHINGTON UNIVERSITY}

HARVARD UNIVERSITY 



\title{
PACIFIC JOURNAL OF MATHEMATICS
}

\section{EDITORS}

\author{
Herbert BuSEMANN \\ R. M. RoBINSON \\ University of Southern California \\ University of California \\ Los Angeles 7, California \\ Berkeley 4, California \\ E. F. BEC KENBACH, Managing Editor \\ University of California \\ Los Angeles 24, California
}

\section{ASSOCIATE EDITORS}
R. P. DILWORTH
P. R. HALMOS
BØRGE JESSEN
J. J. STOKER
HERBERT FEDERER
HEINZ HOPF
PAUL LÉVY
MARSHALL HALL
R. D. JAMES
GEORGE PÓLYA
E. G. STRAUS
KÖSAKU YOSIDA

\section{SPONSORS}

UNIVERSITY OF BRITISH COLUMBIA

CALIFORNIA INSTITUTE OF TECHNOLOGY

UNIVERSITY OF CALIFORNIA, BERKELEY

UNIVERSITY OF CALIFORNIA, DAVIS

UNIVERSITY OF CALIFORNIA, LOS ANGELES

UNIVERSITY OF CALIFORNIA, SANTA BARBARA

OREGON STATE COLLEGE

UNIVERSITY OF OREGON
UNIVERSITY OF SOUTHERN CALIFORNIA

STANFORD UNIVERSITY

WASHINGTON STATE COLLEGE

UNIVERSITY OF WASHINGTON

AMERICAN MATHEMATICAL SOCIETY

NATIONAL BUREAU OF STANDARDS, INSTITUTE FOR NUMERIGAL ANALYSIS

Mathematical papers intended for publication in the Pacific Journal of Mathematics should be typewritten (double spaced), and the author should keep a complete copy. Manuscripts may be sent to any of the editors. All other communications to the editors should be addressed to the managing editor, E. F. Beckenbach, at the address given above.

Authors are entitled to receive 100 free reprints of their published papers and may obtain additional copies at cost.

The Pacific Journal of Mathematics is published quarterly, in March, June, September, and December. The price per volume (4 numbers) is $\$ 8.00$; single issues, $\$ 2.50$. Spécial price to individual faculty members of supporting institutions and to members of the American Mathematical Society: $\$ 4.00$ per volume; single issues, $\$ 1.25$.

Subscriptions, orders for back numbers, and changes of address should be sent to the publishers, University of California Press, Berkeley 4, California.

UNIVERSITY OF CALIFORNIA PRESS - BERKELEY AND LOS ANGELES 


\section{Pacific Journal of Mathematics}

\section{Vol. 1, No. $2 \quad$ December, 1951}

Tom M. (Mike) Apostol, On the Lerch zeta function ................. 161

Ross A. Beaumont and Herbert S. Zuckerman, A characterization of the subgroups of the additive rationals ....................... 169

Richard Bellman and Theodore Edward Harris, Recurrence times for the Ehrenfest model................................... 179

Stephen P.L. Diliberto and Ernst Gabor Straus, On the approximation of a function of several variables by the sum of functions of fewer

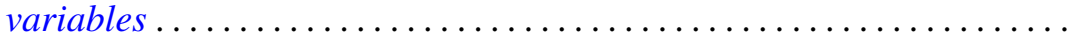

Isidore Isaac Hirschman, Jr. and D. V. Widder, Convolution transforms with complex kernels ................................ 211

Irving Kaplansky, A theorem on rings of operators .............. 227

W. Karush, An iterative method for finding characteristic vectors of a symmetric matrix............................... 233

Henry B. Mann, On the number of integers in the sum of two sets of positive integers ......................................... 249

William H. Mills, A theorem on the representation theory of Jordan

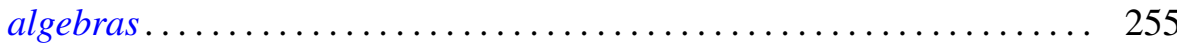

Tibor Radó, An approach to singular homology theory.............. 265

Otto Szász, On some trigonometric transforms ................... 291

James G. Wendel, On isometric isomorphism of group algebras ......... 305

George Milton Wing, On the $L^{p}$ theory of Hankel transforms ... 